\title{
Diphoton production at Hadron Colliders: A fully differential QCD calculation at next-to-next-to-leading order
}

Catani, Stefano ; Cieri, Leandro ; de Florian, Daniel ; Ferrera, Giancarlo ; Grazzini, Massimiliano

\begin{abstract}
We consider direct diphoton production in hadron collisions, and we compute the next-to-nextto-leading order QCD radiative corrections at the fully differential level. Our calculation uses the qT subtraction formalism, and it is implemented in a parton-level Monte Carlo program. The program allows the user to apply arbitrary kinematical cuts on the final-state photons and the associated jet activity and to compute the corresponding distributions in the form of bin histograms. We present selected numerical results related to Higgs boson searches at the LHC and corresponding results at the Tevatron.
\end{abstract}

DOI: https://doi.org/10.1103/PhysRevLett.108.072001

Posted at the Zurich Open Repository and Archive, University of Zurich

ZORA URL: https://doi.org/10.5167/uzh-70202

Journal Article

Originally published at:

Catani, Stefano; Cieri, Leandro; de Florian, Daniel; Ferrera, Giancarlo; Grazzini, Massimiliano (2012). Diphoton production at Hadron Colliders: A fully differential QCD calculation at next-to-next-to-leading order. Physical Review Letters, 108(7):072001.

DOI: https://doi.org/10.1103/PhysRevLett.108.072001 
ZU-TH 18/11

IFUM-984-FT

\title{
Diphoton production at hadron colliders: a fully-differential QCD calculation at NNLO
}

\author{
Stefano Catani ${ }^{(a)}$, Leandro Cieri $^{(b)}$, Daniel de Florian $^{(b)}$, \\ Giancarlo Ferrera $^{(c)}$ and Massimiliano Grazzini ${ }^{(d) *}$ \\ ${ }^{(a)}$ INFN, Sezione di Firenze and Dipartimento di Fisica e Astronomia, \\ Università di Firenze, I-50019 Sesto Fiorentino, Florence, Italy \\ (b) Departamento de Física and IFIBA, FCEYN, Universidad de Buenos Aires, \\ (1428) Pabellón 1 Ciudad Universitaria, Capital Federal, Argentina \\ (c) Dipartimento di Fisica, Università di Milano and \\ INFN, Sezione di Milano, I-20133 Milan, Italy \\ ${ }^{(d)}$ Institut für Theoretische Physik, Universität Zürich, CH-8057 Zürich, Switzerland
}

\begin{abstract}
We consider direct diphoton production in hadron collisions, and we compute the nextto-next-to-leading order (NNLO) QCD radiative corrections at the fully-differential level. Our calculation uses the $q_{T}$ subtraction formalism and it is implemented in a parton level Monte Carlo program. The program allows the user to apply arbitrary kinematical cuts on the final-state photons and the associated jet activity, and to compute the corresponding distributions in the form of bin histograms. We present selected numerical results related to Higgs boson searches at the LHC and corresponding results at the Tevatron.
\end{abstract}

October 2011

*On leave of absence from INFN, Sezione di Firenze, Sesto Fiorentino, Florence, Italy. 
Diphoton production is a relevant process in hadron collider physics. It is both a classical signal within the Standard Model (SM) and an important background for Higgs and new-physics searches. The origin of the Electroweak symmetry breaking is currently being investigated at the LHC by searching for the Higgs boson and eventually studying its properties. If the mass $m_{H}$ of the Higgs boson is low $\left(m_{H} \lesssim 140 \mathrm{GeV}\right)$, the preferred search mode at the LHC involves Higgs boson production via gluon fusion followed by the rare decay into a pair of photons. Therefore, it is essential to count on an accurate theoretical description of the various kinematical distributions associated to the production of pairs of prompt photons with large invariant mass. Such task requires detailed computations of radiative corrections.

In this paper we are interested in the process $p p \rightarrow \gamma \gamma X$ (and the related process $p \bar{p} \rightarrow \gamma \gamma X$ ), which, at the lowest order, occurs via the quark annihilation subprocess $q \bar{q} \rightarrow \gamma \gamma$. The QCD corrections at the next-to-leading order (NLO) in the strong coupling $\alpha_{\mathrm{S}}$ involve the quark annihilation channel and a new partonic channel, via the subprocess $q g \rightarrow \gamma \gamma q$. These corrections have been computed and implemented in the fully-differential Monte Carlo codes DIPHOX [1], 2gammaMC [2] and MCFM [3]. A calculation that includes the effects of transverse-momentum resummation is implemented in RESBOS [4].

At the next-to-next-to-leading order (NNLO), the $g g$ channel starts to contribute, and the large gluon-gluon luminosity makes this channel potentially sizeable. Part of the contribution from this channel, the so called box contribution, was computed long ago [5] and its size turns out to be comparable to the lowest-order result; for this reason, the box contribution is customarily included in all the NLO computations of diphoton production. The next-order gluonic corrections to the box contribution (which are part of the $\mathrm{N}^{3} \mathrm{LO}$ QCD corrections to diphoton production) were computed in Ref. [2] and found to have a moderate quantitative effect on the result of the 'NLO+box' calculation.

Besides their direct production from the hard subprocess, photons can also arise from fragmentation subprocesses of QCD partons. The computation of fragmentation subprocesses requires (poorly known) non-perturbative information, in the form of parton fragmentation functions of the photon. The complete NLO single- and double-fragmentation contributions are implemented in DIPHOX [1]. The effect of the fragmentation contributions is sizeably reduced by the photon isolation criteria that are necessarily applied in hadron collider experiments to suppress the very large irreducible background (e.g., photons that are faked by jets or produced by hadron decays). The standard cone isolation and the 'smooth' cone isolation proposed by Frixione [6] are two of these criteria. The standard cone isolation is easily implemented in experiments, but it only suppresses a fraction of the fragmentation contribution. The smooth cone isolation (formally) eliminates the entire fragmentation contribution, but its experimental implementation is still in progress.

In this Letter we present the computation of the full NNLO QCD corrections to direct diphoton production in hadron collisions. We consider the inclusive hard-scattering reaction

$$
h_{1}+h_{2} \rightarrow \gamma \gamma+X
$$

where the collision of the two hadrons, $h_{1}$ and $h_{2}$, produces the diphoton system $F \equiv \gamma \gamma$ with high invariant mass $M_{\gamma \gamma}$. The evaluation of the NNLO corrections to the process in Eq. (1) requires the knowledge of the corresponding partonic scattering amplitudes with $X=2$ partons (at the tree level [7]), $X=1$ parton (up to the one-loop level [8]) and no additional parton (up to the 
two-loop level [9]) in the final state. The implementation of the separate scattering amplitudes in a complete NNLO (numerical) calculation is severely complicated by the presence of infrared (IR) divergences that occur at intermediate stages. The $q_{T}$ subtraction formalism [10] is a method that handles and cancels these unphysical IR divergences up to the NNLO. The formalism applies to generic hadron collision processes that involve hard-scattering production of a colourless high-mass system $F$. Within that framework [10], the corresponding cross section is written as:

$$
d \sigma_{(N) N L O}^{F}=\mathcal{H}_{(N) N L O}^{F} \otimes d \sigma_{L O}^{F}+\left[d \sigma_{(N) L O}^{F+\text { jets }}-d \sigma_{(N) L O}^{C T}\right]
$$

where $d \sigma_{(N) L O}^{F+\text { jets }}$ represents the cross section for the production of the system $F$ plus jets at $(\mathrm{N}) \mathrm{LO}$ accuracy $^{\dagger}$, and $d \sigma_{(N) L O}^{C T}$ is a (IR subtraction) counterterm whose explicit expression [12] is obtained from the resummation program of the logarithmically-enhanced contributions to $q_{T}$ distributions. The 'coefficient' $\mathcal{H}_{(N) N L O}^{F}$, which also compensates for the subtraction of $d \sigma_{(N) L O}^{C T}$, corresponds to the $(\mathrm{N}) \mathrm{NLO}$ truncation of the process-dependent perturbative function

$$
\mathcal{H}^{F}=1+\frac{\alpha_{\mathrm{S}}}{\pi} \mathcal{H}^{F(1)}+\left(\frac{\alpha_{\mathrm{S}}}{\pi}\right)^{2} \mathcal{H}^{F(2)}+\ldots
$$

The NLO calculation of $d \sigma^{F}$ requires the knowledge of $\mathcal{H}^{F(1)}$, and the NNLO calculation also requires $\mathcal{H}^{F(2)}$.

The general structure of $\mathcal{H}^{F(1)}$ is explicitly known [13]: $\mathcal{H}^{F(1)}$ is directly obtained from the process-dependent scattering amplitudes by using a process-independent relation. Exploiting the explicit results of $\mathcal{H}^{F(2)}$ for Higgs [10, 14] and vector boson [15] production, we have generalized the process-independent relation of Ref. [13] to the calculation of the NNLO coefficient $\mathcal{H}^{F(2)}$ (this general result is presented in a forthcoming paper). Using this relation and the relevant scattering amplitudes $[7,8,9]$, we have explicitly determined $\mathcal{H}^{F(2)}$ for diphoton production.

We have performed our fully-differential NNLO calculation of diphoton production according to Eq. (2). The NNLO computation is encoded in a parton level Monte Carlo program, in which we can implement arbitrary IR safe cuts on the final-state photons and the associated jet activity. The present formulation of the $q_{T}$ subtraction formalism [10] is restricted to the production of colourless systems $F$ and, hence, it does not treat parton fragmentation subprocesses (here $F$ includes one or two coloured partons that fragment). Therefore, we concentrate on the direct production of diphotons, and we rely on the smooth cone isolation criterion [6]. Considering a cone of radius $r=\sqrt{(\Delta \eta)^{2}+(\Delta \phi)^{2}}$ around each photon, we require that the total amount of hadronic (partonic) transverse energy $E_{T}$ inside the cone is smaller than $E_{T \max }(r)$,

$$
E_{T \max }(r) \equiv \epsilon_{\gamma} p_{T}^{\gamma}\left(\frac{1-\cos r}{1-\cos R}\right)^{n}
$$

where $p_{T}^{\gamma}$ is the photon transverse momentum; the isolation criterion $E_{T}<E_{T \text { max }}(r)$ has to be fulfilled for all cones with $r \leq R$. The isolation parameters are set to the values $\epsilon_{\gamma}=0.5, n=1$ and $R=0.4$ in all the numerical results presented in this Letter. We use the Martin-StirlingThorne-Watt (MSTW) 2008 [16] sets of parton distributions, with densities and $\alpha_{\mathrm{S}}$ evaluated at each corresponding order (i.e., we use $(n+1)$-loop $\alpha_{\mathrm{S}}$ at $\mathrm{N}^{n} \mathrm{LO}$, with $\left.n=0,1,2\right)$, and we consider $N_{f}=5$ massless quarks/antiquarks and gluons in the initial state. The default renormalization

\footnotetext{
${ }^{\dagger}$ In the case of diphoton production, the NLO calculation of $d \sigma_{N L O}^{\gamma \gamma+\text { jets }}$ was performed in Ref. [11].
} 
$\left(\mu_{R}\right)$ and factorization $\left(\mu_{F}\right)$ scales are set to the value of the invariant mass of the diphoton system, $\mu_{R}=\mu_{F}=M_{\gamma \gamma}$. The QED coupling constant $\alpha$ is fixed to $\alpha=1 / 137$.

We apply typical kinematical cuts [17] that are used by the ATLAS and CMS Collaborations in their Higgs search studies. We require the harder photon to have a transverse momentum $p_{T}^{\text {harder }} \geq 40 \mathrm{GeV}$, while for the softer photon we demand $p_{T}^{\text {softer }} \geq 25 \mathrm{GeV}$. The rapidity of both photons is restricted to $\left|y_{\gamma}\right| \leq 2.5$, and the invariant mass of the diphoton system is constrained to lie in the range $20 \mathrm{GeV} \leq M_{\gamma \gamma} \leq 250 \mathrm{GeV}$.

\begin{tabular}{|c|c|c|c|}
\hline$\sigma(\mathrm{fb})$ & LO & NLO & NNLO \\
\hline \hline$\mu_{F}=\mu_{R}=M_{\gamma \gamma} / 2$ & $5045 \pm 1$ & $26581 \pm 23$ & $45588 \pm 97$ \\
\hline$\mu_{F}=\mu_{R}=M_{\gamma \gamma}$ & $5712 \pm 2$ & $26402 \pm 25$ & $43315 \pm 54$ \\
\hline$\mu_{F}=\mu_{R}=2 M_{\gamma \gamma}$ & $6319 \pm 2$ & $26045 \pm 24$ & $41794 \pm 77$ \\
\hline
\end{tabular}

Table 1: Cross sections for $p p \rightarrow \gamma \gamma+X$ at the $L H C(\sqrt{s}=14 \mathrm{TeV})$. The applied cuts are described in the text.

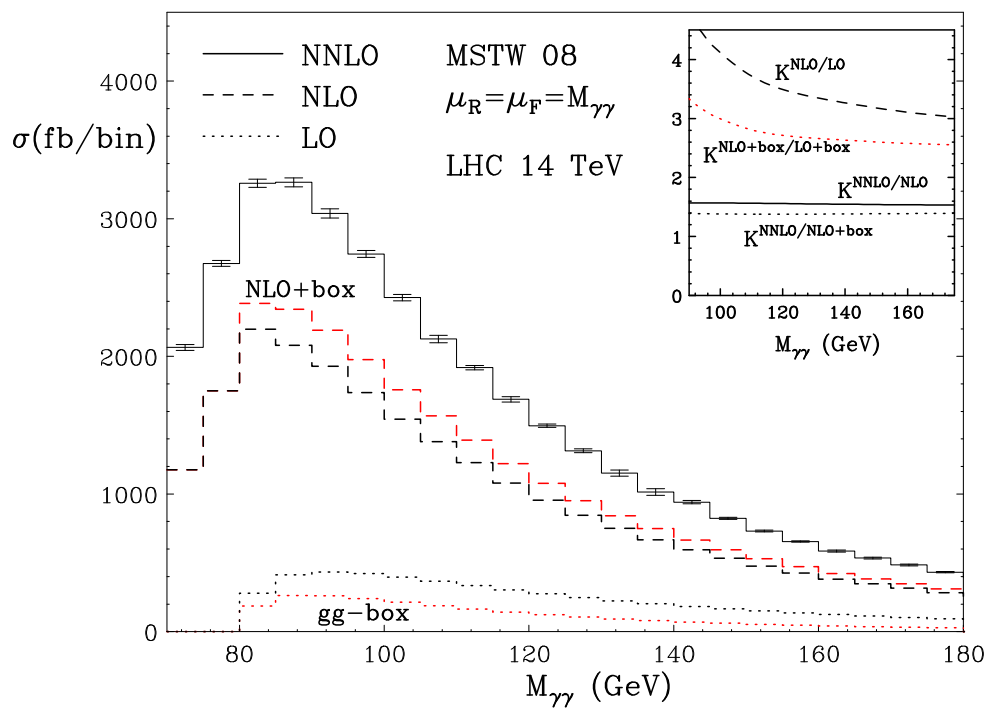

Figure 1: Invariant mass distribution of the photon pair at the $L H C(\sqrt{s}=14 \mathrm{TeV}): L O$ (dots), $N L O$ (dashes) and NNLO (solid) results. We also present the results of the box and $N L O+b o x$ contributions. The inset plot shows the corresponding K-factors.

We start the presentation of our results by considering diphoton production at the LHC $(\sqrt{s}=$ $14 \mathrm{TeV}$ ). In Table 1, we report the results of the accepted cross section at LO, NLO and NNLO. We have fixed $\mu_{F}=\mu_{R}=\mu$ and we have considered three values of $\mu / M_{\gamma \gamma}\left(\mu / M_{\gamma \gamma}=1 / 2,1,2\right)$. The numerical errors estimate the statistical uncertainty of the Monte Carlo integration.

We note that the value of the cross section remarkably increases with the perturbative order of the calculation. This increase is mostly due to the use of very asymmetric (unbalanced) cuts on the photon transverse momenta. At the LO, kinematics implies that the two photons are produced with equal transverse momentum and, thus, both photons should have $p_{T}^{\gamma} \geq 40 \mathrm{GeV}$. 
At higher orders, the final-state radiation of additional partons opens a new region of the phase space, where $40 \mathrm{GeV} \geq p_{T}^{\text {softer }} \geq 25 \mathrm{GeV}$. Since photons can copiously be produced with small transverse momentum (see also Fig. 2 and the related discussion), the cross section receives a sizeable contribution from the enlarged phase space region. This effect is further enhanced by the opening of a new large-luminosity partonic channel at each subsequent perturbative order. For example, at NLO the $q g$ channel accounts for about $80 \%$ of the increase of the cross section. Therefore, it is not unexpected that a naive analysis of scale dependence (as presented in Table 1) underestimates the size of the higher-order corrections.

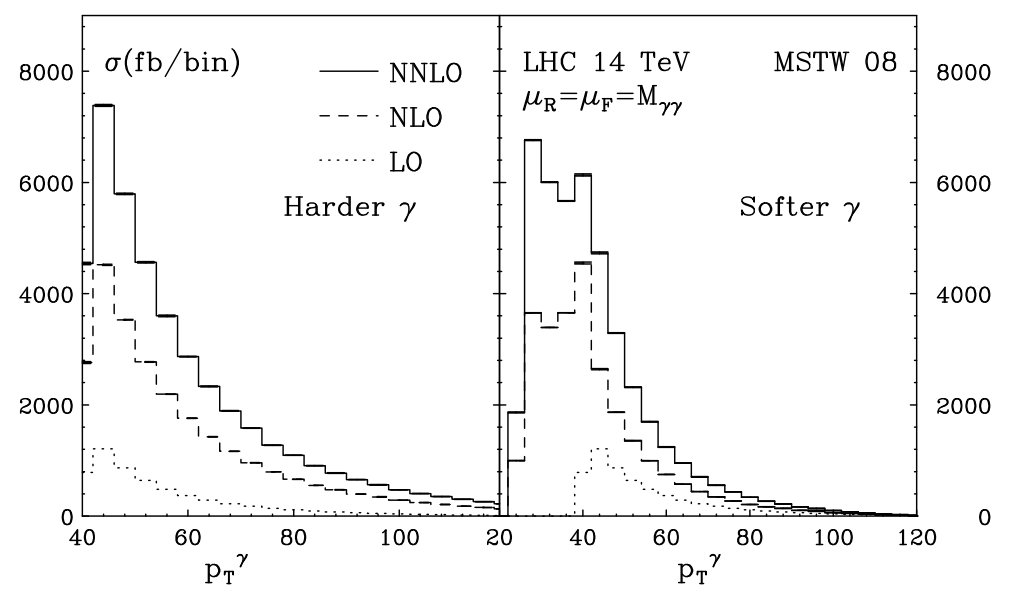

Figure 2: Transverse-momentum distribution of the harder (left) and softer (right) photon at the $L H C(\sqrt{s}=14 \mathrm{TeV})$.

In Figure 1 we compare the LO, NLO and NNLO invariant mass distributions at the default scales. We also plot the gluonic box contribution (computed with NNLO parton distributions) and its sum with the full NLO result. The inset plot shows the K-factors defined by the ratio of the cross sections at two subsequent perturbative orders. We note that $\mathrm{K}^{N N L O / N L O}$ is sensibly smaller than $\mathrm{K}^{N L O / L O}$, and this fact indicates an improvement in the convergence of the perturbative expansion. In particular, the impact of the full NNLO corrections turns out to be reasonably moderate, with a K-factor, defined as the ratio between the NNLO and NLO+box distributions, of about $\mathrm{K} \simeq 1.35$. We find that about $30 \%$ of the NNLO corrections is due to the $g g$ channel (the box contribution is responsible for more than half of it), while almost $60 \%$ still arises from the next-order corrections to the $q g$ channel. The NNLO calculation includes the perturbative corrections from the entire phase space region (in particular, the next-order correction to the dominant $q g$ channel) and the contributions from all possible partonic channels (in particular, a fully-consistent treatment of the box contribution to the $g g$ channel $\left.^{\ddagger}\right)$. Owing to these reasons, the NNLO result can be considered a reliable estimate of direct diphoton production, although further studies (including independent variations of $\mu_{R}$ and $\mu_{F}$, and analyses of kinematical distributions) are necessary to quantify the NNLO theoretical uncertainty.

In Fig. 2 we show results on more exclusive observables: the $p_{T}$ distributions of the harder (left-hand plot) and softer (right-hand plot) photon. The statistical errors of the Monte Carlo

${ }^{\ddagger}$ The calculation [2] of the next-order gluonic corrections to the box contribution indicates an increase of the NNLO result by less than $10 \%$ if $M_{\gamma \gamma} \gtrsim 100 \mathrm{GeV}$. 
integration are at the per-cent level and hardly visible in Fig. 2. As previously anticipated, in the right-hand plot we observe that significant NLO and NNLO contributions to the cross section originate from the phase space region $\left(25 \mathrm{GeV} \leq p_{T}^{\text {softer }} \leq 40 \mathrm{GeV}\right)$ that, at LO, is kinematically forbidden by the asymmetric transverse-momentum cuts. In this low- $p_{T}$ region, the production mechanism of the softer photon is dynamically enhanced (the production probability is roughly proportional to $\alpha_{\mathrm{S}} \times\left(p_{T}^{\text {harder }} / p_{T}^{\text {softer }}\right) \ln \left(p_{T}^{\text {harder }} / p_{T}^{\text {softer }}\right)$, if $\left.p_{T}^{\text {softer }} / p_{T}^{\text {harder }} \sim\left(p_{T}^{\text {softer }} / M_{\gamma \gamma}\right)^{2} \ll 1\right)$, and this is responsible for a substantial part of the large higher-order corrections observed in Table 1, Fig. 1 and also in the $p_{T}$ distribution of the harder photon. Comparing the $p_{T}$ distributions of the harder and softer photon in the high- $p_{T}$ region $\left(p_{T} \gtrsim 50 \mathrm{GeV}\right)$, we also observe that the distribution of the softer photon receives higher-order corrections that are sensibly smaller. This decrease is expected: if both photons have high $p_{T}$, the effect of the very asymmetric transverse-momentum cuts is reduced.

We also comment on the $p_{T}$ distribution of the softer photon in the region around the LO threshold $\left(p_{T}^{\text {softer }} \sim 40 \mathrm{GeV}\right)$. Here the LO result has a step-like behaviour, and this necessarily produces [18] integrable logarithmic singularities at each subsequent perturbative order. The peak of the NLO and NNLO distributions at $p_{T}^{\text {softer }} \sim 40 \mathrm{GeV}$ is an artifact of these perturbative instabilities. The instability is cured by all-order perturbative resummation, which eventually leads to a smooth $p_{T}$ distribution with a shoulder-like behaviour [18] in the vicinity of the LO threshold. This physical behaviour can be approximated (mimicked) in the NLO and NNLO calculations by smearing the distribution over a bin (with a sufficiently-large size) centered around $p_{T}^{\text {softer }} \sim 40 \mathrm{GeV}$.

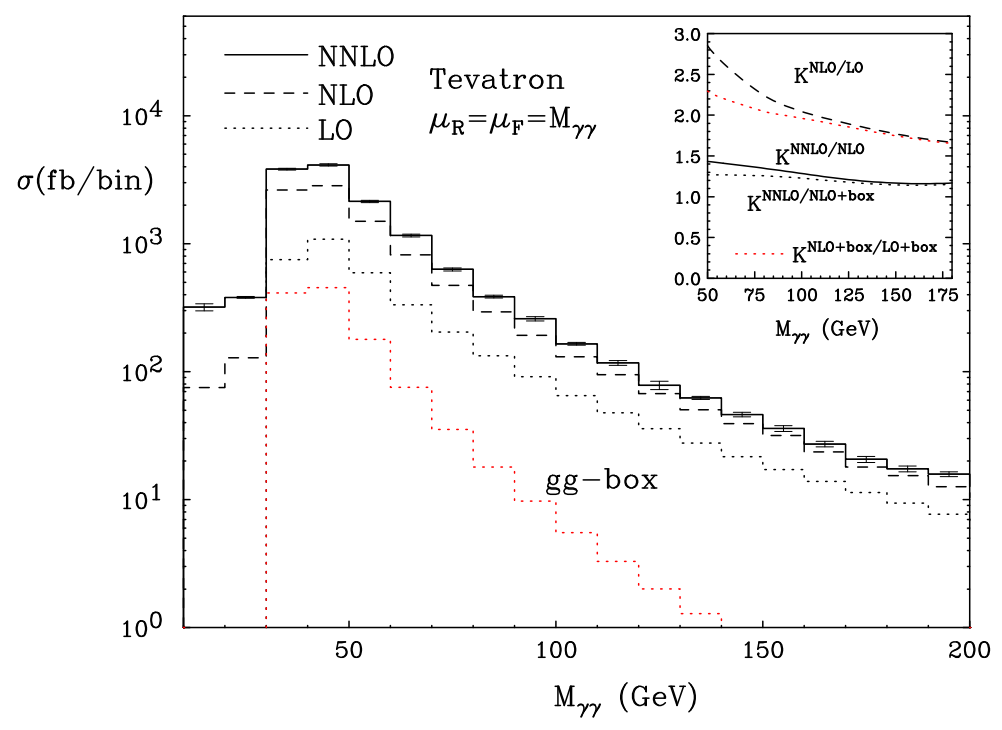

Figure 3: Same as Fig. 1, but for Tevatron kinematics (as described in the text).

In Figure 3, we present the invariant mass distribution for diphoton production at the Tevatron $(\sqrt{s}=1.96 \mathrm{TeV})$. We require the harder and softer photon to have a transverse momentum larger than $17 \mathrm{GeV}$ and $15 \mathrm{GeV}$, respectively. The rapidity of both photons is restricted to $\left|y_{\gamma}\right| \leq 1$. We note that the increase from the LO to the NLO result is considerably smaller than in Fig. 1: this is mostly due to the use of photon transverse-momentum cuts that are only slightly asymmetric. In the region where $M_{\gamma \gamma} \gtrsim 80 \mathrm{GeV}$, the relative impact of the box contribution is smaller than at 
the LHC: this is a consequence of the higher values of parton momentum fractions, $x$, that are probed by Tevatron kinematics. Nevertheless, the NNLO corrections (which are dominated by the next-order correction to the $q g$ channel) still increase the result at the previous order by roughly $30 \%$.

We have presented the calculation of the cross section for diphoton production up to the complete NNLO in QCD perturbation theory. At the NNLO, all the contributions from the $g g$ channel are included in a fully-consistent (and unambiguous) manner. Considering the illustrative isolation and kinematical cuts implemented in this paper, we find increasing effects of about $30 \%-40 \%$ with respect to computations at the previous perturbative order. Our calculation is directly implemented in a parton level Monte Carlo program. This feature makes it particularly suitable for practical applications to the computation of distributions in the form of bin histograms. A public version of our program will become available at a later stage.

Acknowledgements. This work was supported in part by UBACYT, CONICET, ANPCyT, INFN and the Research Executive Agency (REA) of the European Union under the Grant Agreement number PITN-GA-2010-264564 (LHCPhenoNet). We thank the Galileo Galilei Institute for Theoretical Physics for the hospitality during the completion of this work.

\section{References}

[1] T. Binoth, J. P. Guillet, E. Pilon and M. Werlen, Eur. Phys. J. C16, 311 (2000).

[2] Z. Bern, L. J. Dixon and C. Schmidt, Phys. Rev. D66, 074018 (2002).

[3] J. M. Campbell, R. K. Ellis and C. Williams, JHEP 1107, 018 (2011).

[4] C. Balazs, E. L. Berger, P. M. Nadolsky and C. -P. Yuan, Phys. Rev. D76, 013009 (2007).

[5] D. A. Dicus and S. S. D. Willenbrock, Phys. Rev. D37, 1801 (1988).

[6] S. Frixione, Phys. Lett. B429, 369 (1998).

[7] V. D. Barger, T. Han, J. Ohnemus and D. Zeppenfeld, Phys. Rev. D41, 2782 (1990); V. Del Duca, W. B. Kilgore and F. Maltoni, Nucl. Phys. B566, 252 (2000).

[8] Z. Bern, L. J. Dixon and D. A. Kosower, Nucl. Phys. B437, 259 (1995); A. Signer, Phys. Lett. B357, 204 (1995).

[9] C. Anastasiou, E. W. N. Glover and M. E. Tejeda-Yeomans, Nucl. Phys. B629, 255 (2002).

[10] S. Catani and M. Grazzini, Phys. Rev. Lett. 98, 222002 (2007).

[11] V. Del Duca, F. Maltoni, Z. Nagy and Z. Trocsanyi, JHEP 0304, 059 (2003).

[12] G. Bozzi, S. Catani, D. de Florian and M. Grazzini, Nucl. Phys. B737, 73 (2006).

[13] D. de Florian and M. Grazzini, Phys. Rev. Lett. 85, 4678 (2000), Nucl. Phys. B616, 247 (2001). 
[14] S. Catani and M. Grazzini, report ZU-TH-12-11 (arXiv:1106.4652 [hep-ph]).

[15] S. Catani, L. Cieri, G. Ferrera, D. de Florian and M. Grazzini, Phys. Rev. Lett. 103, 082001 (2009).

[16] A. D. Martin, W. J. Stirling, R. S. Thorne and G. Watt, Eur. Phys. J. C63, 189 (2009).

[17] ATLAS collaboration, ATLAS detector and physics performance, technical design report, vol. 2, report CERN/LHCC 99-15, ATLAS-TDR-15; CMS collaboration, CMS: The electromagnetic calorimeter, technical design report, report CERN/LHCC 97-33, CMS-TDR-4.

[18] S. Catani and B. R. Webber, JHEP 9710, 005 (1997). 